\title{
METHODOLOGY
}

\section{Evidence-Based Medicine in Practice: Applying Intention-to-Treat Analysis and Perprotocol Analysis}

Philip S. Schoenfeld, M.D., M.S.Ed., M.Sc. (Epi)

Division of Gastroenterology, University of Michigan, School of Medicine, Ann Arbor, Michigan

\section{CASE SCENARIO}

A 36-yr-old woman with diarrhea-predominant irritable bowel syndrome (IBS) is referred for treatment by her primary care physician. She notes abdominal cramping, bloating, and intermittent loose, watery bowel movements. Over the past 3 months, the intensity and frequency of these symptoms has increased, although she has had similar symptoms intermittently for the past 3-4 yr. She has taken loperamide and fiber products with minimal improvement. Also, the patient denies any alarm symptoms such as hematochezia, weight loss, or nocturnal diarrhea. There is no family history of inflammatory bowel disease or colon cancer, no recent travel, and no fever. Her laboratory tests reveal a normal complete blood cell count and a normal erythrocyte sedimentation rate. Fecal occult blood testing is negative.

\section{CLINICAL QUESTION}

How effective are tricyclic antidepressants at producing global symptom improvement in IBS patients?

\section{EVIDENCE-BASED MEDICINE APPROACH}

A 12-wk, double-blind, randomized controlled trial of 216 patients (1) compared desipramine, a tricyclic antidepressant, versus placebo for global symptom improvement in patients with moderate to severe functional bowel disorders (i.e., abdominal pain with or without altered bowel habit). This study demonstrated no significant global symptom improvement with desipramine versus placebo in the intentionto-treat (ITT) analysis (responder rate: $60 \% v s 47 \%$, respectively; $p=0.16$ ), but did demonstrate a significant benefit with desipramine versus placebo in the perprotocol analysis (responder rate: $73 \%$ vs $49 \%$, respectively; $p=0.01$ ).

\section{TERMINOLOGY}

ITT analysis dictates that all randomized patients are included in the final analysis of data, regardless of whether or not they completed the intervention (2). For example, if a desipramineusing patient in the cited trial (1) discontinues treatment after 3 days because of constipation, dry mouth, and disturbed sleep, then this patient is classified as a treatment failure (i.e., no global symptom improvement) and is included in the final data analysis. In a perprotocol analysis, only randomized patients who complete study therapy are included in the final data analysis. Therefore, our desipramine-using patient who stopped treatment due to side effects would be excluded from the analysis.

Robust studies provide the ITT analysis and the perprotocol analysis. An ITT analysis estimates the likelihood of a successful response to treatment when you start treatment (accounting for the possibility that a patient may discontinue treatment due to lack of efficacy or side effects). A perprotocol analysis provides a best case scenario about the efficacy of a medication, estimating the likelihood of a successful response to treatment if a patient can complete the course of therapy. Therefore, the perprotocol analysis may provide an inflated estimate about the efficacy of treatment because patients who discontinue treatment due to side effects are eliminated from data analysis.

\section{APPLICATION OF ITT ANALYSIS AND PERPROTOCOL ANALYSIS TO THE CLINICAL QUESTION}

In the randomized controlled trial comparing desipramine versus placebo in functional bowel disorder patients (1), 32\% (40/135) of desipramine-using patients did not complete the 12 -wk trial. The most common reasons were adverse events $(56 \%, 23 / 40)$ or dissatisfaction with treatment $(20 \%, 8 / 40)$. Conversely, only $16 \%$ (11/66) of placebo-using patients discontinued treatment early with the most common reasons for discontinuation being adverse events $(28 \%, 3 / 11)$ and dissatisfaction with treatment $(28 \%, 3 / 11)$. Therefore, it is not surprising that the ITT analysis demonstrates a smaller, nonsignificant global symptom improvement with desipramine versus placebo (responder rate: $60 \%$ vs $47 \%$, respectively; $p=0.016)$ compared to the perprotocol analysis, which demonstrates a large and statistically significant improvement with desipramine versus placebo (responder rate: $73 \%$ vs $49 \%$, respectively; $p=0.01$ ). So, if a patient can complete $12 \mathrm{wk}$ of therapy with relatively high doses of desipramine without clinically important side effects or poor therapeutic response, then desipramine-using patients are more likely to benefit than patients receiving placebo. Nevertheless, over 
$30 \%$ of desipramine-using patients will discontinue medication due to side effects or inadequate results.

\section{APPLYING RESULTS TO YOUR PATIENT}

Given the large drop out of desipramine-using patients due to side effects, can we identify the patients who are most likely to tolerate and benefit from desipramine? Twenty-six percentage of the desipramine-using patients complained of constipation compared with only $9 \%$ of placebo-using patients. Drossman et al. (1) prospectively planned subgroup analysis of diarrhea-predominant IBS patients versus constipationpredominant IBS patients. Notably, they demonstrated a statistically significant increase in global symptom improvement among diarrhea-predominant IBS patients in the ITT analysis, but there was no benefit among patients who had constipation-predominant IBS. In other words, the constipation side effect of tricyclic antidepressants may actually be beneficial for patients with diarrhea-predominant IBS, while tricyclic antidepressants exert their primary therapeutic effect by minimizing the patient's experience of distention and discomfort in their abdomen through their effect on pain centers in the brain.

\section{BOTTOM LINE}

In my practice, I usually prescribe tricyclic antidepressants as initial therapy for diarrhea-predominant IBS because tricyclic antidepressants improve global symptoms by minimizing the experience of abdominal discomfort and distention and these agents may decrease stool frequency, too. Based on my clinical experience and randomized controlled trials (3), loperamide decreases stool frequency and improves stool consistency in patients with diarrhea-predominant IBS, so I usually supplement tricyclic antidepressants with prn use of loperamide. However, I use much lower dosages of de- sipramine compared to those utilized in the trial by Drossman et al. (1). The authors used $50 \mathrm{mg}$ of desipramine nightly for $1 \mathrm{wk}$, then $100 \mathrm{mg}$ nightly for $1 \mathrm{wk}$, then $150 \mathrm{mg}$ nightly for $10 \mathrm{wk}$. This rapid increase in desipramine dosage may have increased the frequency of side effects reported in the trial. I slowly escalate the dose of tricyclic antidepressants: $25 \mathrm{mg}$ of desipramine at bedtime with gradual elevation of the dosage to 50-100 mg. I also spend additional time with my IBS patients and explain that I am using tricyclic antidepressants as a treatment for chronic pain and I emphasize that I am not prescribing this medication for depression. If a diarrheapredominant patient primarily complains of stool frequency and stool urgency with minimal abdominal discomfort, then I may start with loperamide before initiating therapy with a tricyclic antidepressant.

I do not routinely recommend tricyclic antidepressants for patients with nondiarrhea predominant IBS. Multiple large randomized controlled trials in nondiarrhea predominant IBS patients demonstrate that tegaserod $6 \mathrm{mg}$ b.i.d. is significantly better than placebo at improving global IBS symptoms, bloating, and abdominal discomfort (3).

\section{REFERENCES}

1. Drossman BA, Toner BB, Whitehead WE, et al. Cognitive behavioral therapy versus education and desipramine versus placebo for moderate to severe functional bowel disorders. Gastroenterology 2003;125:19-31.

2. Schoenfeld P, Cook D, Hamilton S, et al. An evidencebased approach to gastroenterology therapy. Gastroenterology 1999;116:1230-37.

3. American College of Gastroenterology Functional GI Disorders Task Force. Evidence-based approach to the management of irritable bowel syndrome. Am J Gastroenterol 2002;97:S1-26.

Reprint requests and correspondence: Philip Schoenfeld, M.D., VAMC 111-D, 2215 Fuller Road Ann Arbor, MI, 48105. 